\title{
A qualidade de vida e o comportamento otimista de pessoas com HIV/AIDS
}

\section{The quality of life and the optimistic behavior of people with HIV/AIDS}

\author{
Bruno José Frederico Pimenta \\ Universidade Estadual de Londrina - UEL - Londrina - Paraná - Brasil \\ jb.frederico@gmail,com \\ Camila Ramos dos Santos \\ Universidade Estadual de Londrina - UEL - Londrina - Paraná - Brasil \\ camilaramos 3@,hotmail.com \\ Lenamar Fiorese Vieira \\ Universidade Estadual de Maringá - UEM - Maringá - Paraná - Brasil \\ lenamarfiorese@gmail.com \\ Inara Marques \\ Universidade Estadual de Londrina - UEL - Londrina - Paraná - Brasil \\ inaramarques@hotmail.com
}

\section{RESUMO}

OBJETIVO: Avaliar a relação entre o otimismo e os domínios de qualidade de vida em pessoas vivendo com Human Immunodeficiency Virus (HIV)/Síndrome da Imunodeficiência Adquirida (AIDS) (PVHA).

MÉTODOS: Participaram deste estudo 15 indivíduos soropositivos, assistidos por uma Organização não Governamental de Redução de Danos. Os instrumentos de avaliação foram uma anamnese para caracterização dos participantes, o questionário de otimismo em eventos futuros e o questionário de qualidade de vida (SF-36). A análise dos dados foi conduzida por meio da estatística descritiva, mediana e intervalo interquartil e Correlação de Spearman adotando $p<0,05$.

RESULTADOS: Constatou-se baixo nível de otimismo dos sujeitos e nível moderado de qualidade de vida. Não houve correlações significativas entre otimismo e os domínios de qualidade de vida do SF-36.

CONCLUSÃO: Os dados do presente estudo permite concluir que níveis de otimismo não influenciam na autopercepção da qualidade de vida das pessoas com HIV/AIDS que frequentam uma organização não governamental.

PALAVRAS-CHAVE: Otimismo. Qualidade de Vida. AIDS. 


\section{ABSTRACT}

OBJECTIVE: Evaluate the level of relationship between optimism and areas of quality of life in persons living with HIV/AIDS (PLHA).

METHODS: Fifteen persons living with HIV/AIDS of a NGO participate of this study. The valuation tools used were: an anamnesis for characterization the participants, an test of life orientation (TOV-R) and a questionnaire about quality of life (SF-36). The data analyses was conducted using descriptive statistics, median and interquartile range, and Spearman correlation adopting $\mathrm{p}<0,05$.

RESULTS: The results showed low level of optimism of the subject and moderate level of quality of life. There were no significant correlations between optimism and areas of quality of life of SF-36.

CONCLUSIONS: The data from this study shows that optimism levels did not influence the self-perception of the quality of life of people with HIV/AIDS who frequent a non-governmental organization.

KEYWORDS: Optimism. Quality of life. AIDS.

\section{Introdução}

O Human Immunodeficiency Virus - Vírus da Imunodeficiência Humana (HIV) ou a Síndrome da Imunodeficiência Adquirida (AIDS) atinge aproximadamente 700 mil brasileiros e tem apresentado uma tendência de aumento, principalmente nas regiões norte e nordeste do país. Atualmente, as medidas profiláticas adotadas pelo governo brasileiro para tentar conter o curso da infecção têm se caracterizado pela identificação precoce da condição sorológica do infectado e, posteriormente, a sua imediata inserção na Terapia Anti-Retroviral (TARV) (BRASIL, 2014).

O tratamento da infecção tem sido feita por meio da TARV que é caracterizada, geralmente, por três classes de retrovirais. Os retrovirais agem no controle da replicação viral e na manutenção das células $\mathrm{CD} 4+$, o que faz postergar o surgimento do(a) HIV/AIDS e dificulta surgimento de infecções oportunistas (ELLIS; CALERO; STOCKIN, 2009). Desde 1996, a distribuição de medicamentos retrovirais tem sido feita gratuitamente pelo governo brasileiro por meio do Sistema Único de Saúde (SUS), o que tem garantido a universalização do tratamento para toda a população brasileira (BRASIL, 1996). O acesso universal à TARV possibilitou o aumento da expectativa de vida das pessoas que vivem com HIV/AIDS (PVHA) (DEEKS; LEWIN; HAVLIR, 2013), no entanto, mudanças têm sido observadas no perfil de morbi-mortalidade da epidemia no Brasil (DOURADO et al., 2006).

As mudanças ocorridas no curso da infecção com a introdução da TARV (ROSSI; MALUF; CARVALHO, 2012) faz com que as PVHA estejam expostas por mais tempo as toxidades da medicação (BUTT et al., 2004) e as ações diretas do HIV que agem, principalmente, no Sistema Nervoso Central (SNC), desencadeando complicações corticais e subcorticais (CHRISTO, 2010). Essa nova condição do infectado tem, em muitos casos, desencadeado comorbidades como a diabetes (BUTT et al, 2004) e distúrbios neurológicos de ordem motora, cognitiva e comportamental (CHRISTO, 2010).

As comorbidades têm se evidenciado nos mais diversos graus e são, mais frequentemente, observados conforme a doença progride para os estágios mais avançados (CHRISTO, 2010; TRENKWALDER et al., 1992). Concomitante a esses 
casos de prevalência e incidência de distúrbios de ordem fisiológica, os soropositivos, além das barreiras sociais, que estão relacionadas aos estigmas e preconceitos socialmente impostos (MEIRELES et al., 2010), enfrentam ainda barreiras psicológicas, que são mais visíveis, principalmente, no momento em que se recebe o diagnóstico de sorologia positiva (REIS, 2008).

Percebe-se, então, que esse amplo número de variáveis (físiológicas, sociais, psicológicas) podem, de forma direta ou indireta, comprometer a autopercepção da qualidade de vida por parte das PVHA (REIS, 2008). Entretanto, a pouco mais de duas décadas, a psicologia tem observado que um comportamento otimista pode ser determinante para que eventos estressores, como os vividos pelas PVHA, sejam superados (SELIGMAN; CSIKSZENTMIHALYI, 2000), mesmo que ainda não se tenha muita informação acerca do real impacto de um comportamento otimista/pessimista sobre a autopercepção da qualidade de vida, justificando, assim, a necessidade de mais estudos (MOYER et al., 2008).

O constructo otimismo tem sido mensurado segundo as expectativas favoráveis ao futuro que um indivíduo possui (BANDEIRA et al., 2002). As evidências empíricas envolta dessa abordagem positivista da psicologia têm mostrado que as pessoas com características otimistas apresentam medidas proativas de engajamento, enfrentamento e, consequentemente, podem apresentar maiores níveis de autopercepção da qualidade de vida em momentos de dificuldades e estresse (CARVER; SCHEIER; SEGERSTROM, 2010).

Os benefícios conferidos pelo otimismo quando comparado ao pessimismo têm sido explicados pela forma distinta com que pessoas lidam e interpretam as situações estressoras (SCHEIER; CARVER, 1992). Neste contexto, constructos como otimismo e pessimismo passaram a ser objetos de estudos na compreensão da sua influência sobre o comportamento humano (SCHEIER; CARVER, 1992).

Essas diferentes formas de enfrentamento das adversidades, aparentemente, influenciam o indivíduo sobre a sua percepção quanto à sua posição na vida, no contexto da cultura e dos sistemas de valores nos quais vive em relações à expectativa, padrões e preocupações (SCHEIER; CARVER, 1992; CARVER; SCHEIER; SEGERSTROM, 2010). Neste contexto, em um dos poucos estudos que investigaram o impacto do otimismo sobre o comportamento de PVHA (TAYLOR et al., 1992), foi relacionado o otimismo com o comportamento sexual de risco e sugeriu-se que não há relação, uma vez que a variável otimismo aparenta ser extremamente adaptativa.

Pelo exposto, nota-se que o entrelaçamento entre variáveis psicológicas, como o otimismo e a autopercepção da qualidade de vida, ainda apresentam divergência na literatura. Isto, principalmente, em populações com doenças crônicas como os infectados pelo HIV (MOYER et al., 2008).

Portanto, o objetivo do presente estudo é avaliar a relação entre o otimismo e os domínios da qualidade de vida em PVHA. Tem-se como pressuposto que um comportamento otimista pode favorecer positivamente na autopercepção sobre a qualidade de vida.

\section{Métodos}

O presente estudo utilizou-se de um corte transversal. Os participantes, todos diagnosticados com HIV/AIDS, foram escolhidos por conveniência em uma Organização Não Governamental (ONG), que está baseada na política de redução de danos voltada para a diminuição da incidência da infecção pelo HIV e outras Doenças Sexualmente Transmissíveis (DSTs). A abordagem destes indivíduos ocorreu durante as 
reuniões de ajuda mútua que ocorrem semanalmente na ONG. Os participantes foram esclarecidos sobre os procedimentos e os objetivos do estudo e, havendo a concordância, assinaram o Termo de Consentimento Livre e Esclarecido (TCLE). O presente estudo foi submetido à apreciação do Comitê de Ética da Universidade local, sob o número 025/2014 o qual foi aprovado respeitando, desta forma, a resolução de número 196/96 do Conselho Nacional de Saúde.

A qualidade de vida foi avaliada por meio do Questionário Genérico de Avaliação da Qualidade de Vida SF-36 (CICONELLI et al., 1999). O questionário SF36 é de fácil administração e compreensão, considerando a percepção dos indivíduos quanto ao seu próprio estado de saúde contemplando os aspectos mais representativos. Com característica multidimensional, o instrumento contém 36 questões sobre os domínios: capacidade funcional; aspecto físico; dor; estado geral de saúde; vitalidade; aspectos sociais; aspectos emocionais e saúde mental. Cada domínio que compõe o SF36 possui escore único e independente dos demais domínios, sendo que o escore final pode variar entre 0 e 100 e, quanto mais próximo de 100 , melhor é a qualidade de vida autopercebida.

A avaliação do otimismo foi feita por meio do Teste de Orientação de Vida (TOV-R), validado para a população brasileira (BANDEIRA et al., 2002). O TOV-R apresenta-se como um teste curto e homogêneo de característica unidimensional, tendo o otimismo e o pessimismo como dois pólos de uma mesma dimensão. Composto por 10 itens, o indivíduo deve responder os intens em função de uma escala tipo Likert de 0 (discordo totalmente) a 4 (concordo totalmente), sendo que quanto mais próximo de 4 , maior é o grau de otimismo.

A caracterização da população foi feita por meio de um questionário socioeconômico e de uma anamnese. A avaliação socioeconômica do grupo seguiu o Critério de Classificação Econômica Brasil (ASSOCIAÇÃO BRASILEIRA DE EMPRESAS DE PESQUISA, 2014), o qual classifica a população em: A1, A2, B1, B2, C1, C2, D e E, conforme o poder de compra, abandonando a pretensão de classificar a população em termos de classes sociais. O questionário da Associação Brasileira de Empresas de Pesquisa (ABEP) é um instrumento simples que avalia a posse de 10 itens: rádio, televisor, automóvel, banheiro, $\mathrm{DVD}$, geladeira ou freezer, mensalista, máquina de lavar e grau de instrução.

Por fim, a anamnese foi composta por questões tais como: tempo de infecção, tempo de uso da TARV e a idade.

Como procedimento de análise, a idade, o tempo de infecção pelo HIV, o tempo de utilização da TARV e o tempo de participação na ONG, foram apresentadas a partir da estatísitca descritiva média e desvio padrão. Por outro lado, devido aos dados não apresentarem uma distribuição normal quando aplicado o teste Shapiro Wilk, as variáveis psicológicas do SF-36 e do TOV-R tiveram a estatística descritiva feita por meio da mediana e do primeiro e terceiro intervalo interquartil, obtendo, assim, a pontuação geral em cada domínio da qualidade de vida e do otimismo. Para a correlação entre o otimismo e a qualidade de vida foi utilizado o teste de Spearman adotando significância $5 \%(\mathrm{P}<0,05)$. A análise estatística foi realizada pelo pacote estatístico SPSS 20.0.

\section{Resultados}

O convite de participação foi aceito por 15 sujeitos assistidos pela ONG que foram caracterizados segundo a média de idade, o tempo de infecção, o tempo de uso da TARV (ressaltando que apenas 1 participante não fazia o uso da TARV) e o tempo de 
ONG como mostra a Tabela 1. Os perfis socioeconômicos dos participantes foram: D $(\mathrm{n}=1) ; \mathrm{A} 2(\mathrm{n}=1)$; B2 $(\mathrm{n}=4)$; C2 (n=9) segundo o Critério de Classificação Econômica Brasil (ASSOCIAÇÃO BRASILEIRA DE EMPRESAS DE PESQUISA, 2014).

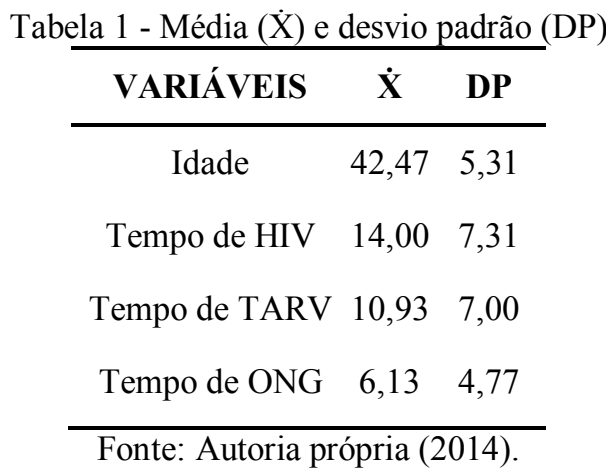

A Tabela 2 apresenta os dados descritivos da mediana e do primeiro e terceiro intervalo interquartil das variáveis psicológicas que compunham o TOV-R e o Questionário Genérico de Avaliação da Qualidade de Vida SF-36.

\begin{tabular}{cccc} 
Tabela 2 - Mediana (Md), primeiro $\left(\mathrm{Q}_{1 / 4}\right)$ & e terceiro $\left(\mathrm{Q}_{3 / 4}\right)$ intervalo \\
\cline { 2 - 5 } VARIÁVEIS & Md & $\mathbf{Q}_{1 / 4}$ & $\mathbf{Q}_{3 / 4}$ \\
\hline Q- Otimismo & 2,5 & 2,3 & 3,1 \\
Capacidade Funcional & 90 & 85 & 95 \\
Limitação por aspectos Físicos & 75 & 12,5 & 75 \\
Dor & 41 & 26 & 100 \\
Estado Geral de Saúde & 52 & 40 & 62 \\
Vitalidade & 65 & 55 & 70 \\
Aspectos Sociais & 75 & 62,5 & 75 \\
Limitação Emocional & 66,6 & 49,6 & 100 \\
Saúde Mental & 72 & 60 & 80 \\
Fonte: Autoria própria $(2014)$. &
\end{tabular}

Os resultados sinalizaram valores médios e de desvio padrão identificando na maioria dos indivíduos que o tempo de descoberta de $\operatorname{HIV~(X:~14,00;~DP:~7,31)~é~bem~}$ próximo do tempo de uso da TARV (X்: 10,93; DP: 7,00), deixando evidente que a maioria das pessoas logo que descobrem a doença começam a fazer uso da medicação.

Outro ponto a ser observado é que de todos os domínios do questionário SF-36, apenas dor apresentou valor menor que 50 pontos (41 pontos), indicando que, mesmo dentro da condição de doença crônica, essa população apresenta níveis de qualidade de vida entre escores moderados (por exemplo, 52 pontos no domínio estado geral de saúde) e altos (por exemplo, 90 pontos no domínio capacidade funcional) (Tabela 2).

Os valores do otimismo foram de medianas baixas considerando que o índice vai até 4,0 pontos.

Os valores de correlação entre o TOV-R com os domínios do Questionário Genérico de Avaliação da Qualidade de Vida SF-36 não apresentaram significância, como pode ser observado na Tabela 3. 
Tabela 3 - Dados das correlações de Spearman

\begin{tabular}{ccccccccc}
\hline & CF & LF & DR & EGS & VT & AS & LAE & SM \\
\hline Otimismo & 0,27 & 0,13 & $-0,07$ & 0,22 & 0,04 & $-0,04$ & $-0,06$ & 0,24 \\
\hline \multicolumn{7}{c}{ Fonte: Autoria própria (2014). }
\end{tabular}

Legenda: CF. Capacidade funcional; LF. Limitação por aspectos físicos; DR. Dor; EGS. Estado geral de saúde; VT. Vitalidade; AS. Aspectos sociais; LE. Limitação por aspecto emocional; SM.

Saúde mental.

A análise entre os domínios do SF-36 apresentam correlações significativas como expressa a Tabela 3 .

Observa-se que a capacidade funcional apresentou correlações de $p=0,62$ (Tabela 4) quando correlacionada com limitações por aspectos físicos. Esse mesmo domínio, também, apontou correlações com dor $(p=0,51)$ (Tabela 4) e com saúde mental $(p=0,53)$ (Tabela 4). Outros domínios correlacionados foram dor com vitalidade, apresentando valores de $p=0,59$ (Tabela 4); aspectos sociais com estado geral de saúde $(p=0,62)$ (Tabela 4) e vitalidade com saúde mental, na qual foram observadas as maiores correlações $(\mathrm{r}=0,78)$ (Tabela 4$)$.

Tabela 4 - Dados das correlações dos domínios do SF-36

\begin{tabular}{|c|c|c|c|c|c|c|c|c|}
\hline & $\mathrm{CF}$ & $\mathrm{LF}$ & DR & EGS & $\mathrm{VT}$ & AS & LAE & SM \\
\hline $\mathrm{CF}$ & ------- & $0,62 *$ & $0,51^{*}$ & 0,25 & 0,39 & 0,20 & 0,33 & $0,53^{*}$ \\
\hline $\mathrm{LF}$ & ------- & -------- & 0,22 & $-0,10$ & 0,07 & $-0,41$ & 0,16 & 0,09 \\
\hline DR & ------- & ------ & -------- & 0,30 & $0,59 *$ & 0,43 & $-0,13$ & 0,21 \\
\hline EGS & ------- & -------- & ------- & ------- & 0,30 & $0,62 *$ & 0,21 & 0,11 \\
\hline VT & -------- & ------ & ------ & ------ & ------- & 0,11 & $-0,14$ & $0,78 * *$ \\
\hline AS & ------- & ------- & ------ & ------ & ----- & --------- & 0,34 & 0,04 \\
\hline LAE & -------- & -------- & ------- & ----- & ----- & ------ & -------- & 0,04 \\
\hline $\mathrm{SM}$ & ------- & ------ & ------- & ----- & ------- & ------ & ------ & - \\
\hline
\end{tabular}

Legenda: CF. Capacidade funcional; LF. Limitação por aspectos físicos; DR. Dor; EGS.

Estado geral de saúde; VT. Vitalidade; AS. Aspectos sociais; LE. Limitação por aspecto emocional; SM. Saúde mental.

$* p<0,05$ com valor de $p=0,51$ a $p=0,62$. ** $p<0,01$ com valor de $p=0,78$

\section{Discussão}

Os principais achados no presente estudo não evidenciaram a correlação entre o otimismo e os domínios de qualidade de vida (Tabela 3). Desta forma, os resultados do presente estudo se opõem aos achados de Wrosh e Scheier (2003), no qual os autores sugerem que as variáveis de qualidade de vida estão relacionadas às características subjetivas de cada indivíduo, como por exemplo, o otimismo que pode atuar na promoção da qualidade de vida. Uma das explicações pode estar no baixo nível de otimismo dos sujeitos.

A adoção de um perfil otimista por parte das PVHA também foi questão de estudo de Taylor et al. (1992). Os resultados desse estudo corroboraram com os achados do presente. Entretanto, diferente desse estudo, Taylor et al. (1992) investigaram a possível correlação entre um perfil otimista e a adoção de um comportamento sexual de risco, concluindo que o otimismo é altamente adaptativo e pode, não necessariamente, afetar o comportamento e as estratégias de enfrentamento frente aos eventos estressores.

Embora não se tenha observado correlação entre otimismo e a qualidade de vida, foi possível observar que as PVHA apresentaram valores moderados e altos de correlação entre os domínios de qualidade de vida (Tabela 4). Isso sugere que as 
variáveis psicológicas e físicas podem ser correlacionadas com a capacidade funcional assim como o contrário também aparenta ser verdadeiro. Essa evidencia pode ser sustentada no estudo de Silva, Saldanha e Azevedo (2010). Nesse estudo, foram observadas as variáveis que exercem impacto sobre qualidade de vida de PVHA. Concluiu-se que as dimensões que compõe o constructo qualidade de vida são indissociáveis.

Ao observar os valores moderados e altos de autopercepção da qualidade de vida (Tabela 2), percebe-se que mesmo frente à heterogenidade das condições clinicas dos participantes deste estudo, a percepção da qualidade de vida tem sido mantida moderada e alta. Esses dados são similares aos achados por outro estudo (SANTOS; FRANÇA JUNIOR; LOPES, 2007) que, ao avaliar a qualidade de vida de uma população com HIV/AIDS heterogênea, observaram que as pessoas com AIDS apresentam uma melhor autopercepção da qualidade de vida que outros pacientes que se encontram em outros estágios da doença. A relação social foi o único domínio em que as pessoas com AIDS tiveram uma menor percepção quando comparado com pessoas em outros estágios da doença. Tal achado difere do presente estudo, uma vez que o domínio aspectos sociais foi o segundo mais bem pontuado (75 pontos) (Tabela 3 ).

A boa pontuação nos aspectos sociais evidencia que as instituições que assistem e promovem o bem-estar psicossocial da população soropositiva tornam-se componente importante na melhora da qualidade de vida (SILVA, 1998). Neste sentido, a ONG na qual os participantes do presente estudo estão inseridos desenvolve reuniões semanais com os assistidos, cujo objetivo é oferecer um processo de politização da AIDS a partir da ajuda ao próximo e das trocas de experiências. A situação vem ao encontro com outro estudo (CASTO-SILVA; HEWITT; CAVICHIOLI, 2007) que comparou ONGs brasileiras e canadenses que assistem soropositivos. Nesse estudo observou-se que o suporte social pode influenciar na forma como são internalizados e percebidos os eventos ocorridos na vida das PVHA.

A alta pontuação conferida à capacidade funcional (90 pontos) merece destaque (Tabela 2), embora possa ser atribuída, em parte, ao uso da TARV (CARDOSO, 2002; TSE et al., 2004). A variável capacidade funcional revela uma correlação com componentes físicos e psicológicos que não foram observados em no estudo de Vidrine et al. (2004). Nesse estudo, os autores associaram variáveis sociodemograficas e comportamentais à qualidade de vida utilizando o SF-12 (uma versão curta do SF-36).

Finalizando, observa-se que a qualidade de vida é um constructo multidimensional que se mostra dependente de variáveis objetivas e subjetivas de cada indivíduo, independente da presença ou não de patologias. Entretanto, embora o impacto das variáveis subjetivas, tais como o otimismo e o pessimismo, possam ser componentes fundamentais para a melhora da autopercepção, o seu impacto sobre outras variáveis que buscam avaliar a qualidade de vida ainda apresentam divergências na literatura.

\section{Considerações finais}

Embora nos últimos anos a psicologia tenha evidenciado a importância de um perfil otimista para superar condições estressoras, o mesmo perfil não se correlacionou com diversos constructos de qualidade de vida das PVHA. Por outro lado, os dados do presente estudo evidenciaram que constructos de qualidade de vida, tais como fatores físicos, saúde mental e capacidade funcional, apresentam correlação entre si, sugerindo que a melhora ou piora destes domínios podem transcender para outras variáveis. Ainda um resultado importante foi o baixo nível de otimismo dos participantes. 
Considerando que parte dos dados do presente estudo permitem a compreensão e melhoria da qualidade de vida das PVHA, compreende-se que os mesmos podem ter sido influenciados por vieses devido à heterogenidade dos participantes, estágio da doença, utilização da TARV e assiduidade aos encontros na ONG. Suger-se que futuros estudos buscando compreender os efeitos do otimismo/pessimismo sobre a qualidade de vida das PVHA concentrem-se numa amostra mais homogênea quanto ao tempo de diagnóstico e à idade dos participantes.

\section{Referências}

ASSOCIAÇÃO BRASILEIRA DE EMPRESAS DE PESQUISA. Critério de classificação econômica. Disponível em: $<\underline{w w w}$.abep.org/criterio-brasil $>$. Acesso em: 16 jun. 2014.

BANDEIRA M.; BEKOU V.; LOTT, K. S.; TEIXEIRA, M. A.; ROCHA, S. S. Validação transcultural do teste de orientação de vida (TOV-R). Estudos de Psicologia, v. 7 , n. 2, p. 251-258, 2002. crossef

BRASIL. Boletim Epidemiológico HIV-AIDS. Brasília: Ministério da Saúde, 2014. Disponível em: <www.aids.gov.br/publicacao/2014/boletim-epidemiologico-2014>. Acesso em: 16 jun. 2014.

BRASIL. Lei n. 9313, de 13 de novembro de 1996. Dispõe sobre a distribuição de medicamentos aos portadores do HIV e doentes de AIDS. Lex: Coleção de Leis do Brasil, Brasília, v. 11, p. 23725, nov., 4 trim., 1996.

BUTT A. A.; FULTZ, S. L.; KWOH, K.; KELLEY, D.; SKANDERSON, M.; JUSTICE, A.; C. Risk of diabetes in HIV infected veterans pre- and post-HAART and the role of HCV coinfection. Hepatology, v. 40, n. 1, p. 115-119, 2004. rossef

CARDOSO, F. HIV-related moviment disorders: epidemiology, pathogenesis and management. CNS Drugs, v. 16, p. 663-668, 2002. Crossef

CARVER, C.; SCHEIER, M. F.; SEGERSTROM, K. S. Optimism. Clinical Psychology Review, v. 30, p. 879-889, 2010. Crossef

CASTRO-SILVA, C. R.; HEWITT, W. E.; CAVICHIOLI, S. Igualdade e dessimetrias: a participação política em ONGs HIV/AIDS do Canadá e do Brasil. Revista Psicologia \& Sociedade, v. 19, n. 2, p. 79-88, 2007. Crossef

CHRISTO, P. P. Alterações cognitivas na infecção pelo HIV e AIDS. Revista da Associação Médica Brasileira, v. 56, n. 2, p. 242-247, 2010. Crossef

CICONELLI, R. M.; FERRAZ, M. B.; SANTOS W.; MEINÃO I.; QUARESMA, M. R. Tradução para a língua português e validação do questionário genérico de avaliação de qualidade de vida SF-36 (Brasil SF-36). Revista Brasileira de Reumatologia, v. 39, p. 143-150, 1999.

DEEKS, S. G.; LEWIN, S. R.; HAVLIR, D. V. The end of AIDS: HIV infection as a chronic disease. Lancet, v. 382, p. 1525-1533, 2013. crossef 
DOURADO, I.; VERSA, M. A. S. M.; BARREIRA, D.; BRITTO, A. M. Tendências da epidemia de AIDS no Brasil após a terapia anti-retroviral. Revista de Saúde Pública, v. 40, p 9-17, 2006. Crossef

ELLIS, R. J.; CALERO, P.; STOCKIN, M. D. HIV infection and the central nervous system: a primer. Neuropsychology Review, v. 19, p. 144-151, 2009. rossef

MEIRELlES, B. H. S.; SILVA, D. M. G. V.; VIEIRA, F. M. A. V.; SOUZA, S. S.; COELHO, I. Z. Percepções da qualidade de vida de pessoas com HIV/AIDS. Revista da Rede de Enfermagem do Nordeste, v. 11, n. 3, p. 68-76, 2010.

MOYER, C. A.; EKPO, G.; CALHOUN, C. L.; GREENE, J.; NAIK, S.; SIPPOLA, E. Qualite of life, optimism/pessimism, and knowledge and attitudes toward HIV screening among pregnant women in Ghana. Womens Health Issues, v. 18, p. 301-309, 2008.

REIS, R. K. Qualidade de vida dos portadores de HIV/AIDS: influência dos fatores demográficos, clínicos e psicossociais. 2008. 274 f. Tese (Doutorado em Enfermagem) - Universidade de São Paulo, Ribeirão Preto, 2008.

ROSSI, S. M. G.; MALUF, C. P.; CARVALHO, D. S.; RIBEIRO, E. L. Impacto da terapia antirretroviral conforme diferentes consensos de tratamento da AIDS no Brasil. Revista Panamericana de Salud Publica, v. 32, p. 117-123, 2012. rossef

SANTOS, E. C. M.; FRANÇA JUNIOR, I.; LOPES, F. Qualidade de vida de pessoas vivendo com HIV/AIDS em São Paulo. Revista de Saúde Pública, v. 41, n. 2, p. 64-71, 2007. crossef

SCHEIER, M. F.; CARVER, C. S. Effects of optimism on psychological and physical well-being: theoretical overview and empirical. Cognitive Therapy and Research, v. 12, p. 201-228, 1992. Crossef

SELIGMAN, M.; CSIKSZENTMIHALYI, M. Positive psychology: an introduction. American Psychologist, v. 55, n. 1, p. 5-14, 2000. Crossef

SILVA, C. L. C. ONGs/AIDS, intervenções sociais e novos laços de solidariedade social. Caderno de Saúde Pública, v. 14, n. 2, p. 129-139, 1998. Crossef

SILVA J.; SALDANHA, A. A. W.; AZEVEDO, R. L. W. Variáveis de impacto na qualidade de vida de pessoas acima de 50 anos HIV+. Psicologia: Reflexão e Crítica, v. 23 , n. 1, p. 56-63, 2010. Crossef

TAYLOR, S. E.; KEMENY, M. E.; APINWALL, L. G.; SCHNEIDER, S. G.; RODRIQUEZ, R.; HEBERT, M. Optimism, coping, psychological distress and highrisk sexual behavior among men at risk acquired immunodeficiency syndrome (AIDS). Journal of Personality and Social Psychology, v. 63, n. 3, p. 460-473, 1992. rossef 
TRENKWALDER, C.; STRABUE, A.; PAULUS, W.; KRAFCZYK, E. S.; EINHIUPL, K. M. Postural imbalance: an early sing in HIV-1 infected patients. European Achives of Pasychiatry and Clinical Neuroscience, v. 241, p. 267-272, 1992. crossef

TSE, W.; CERSOSIMO, M. G.; GRACIES, J. M.; MORGELlO, S.; OLAMOW, C.; KOLLER, W. Moviment disorders and AIDS: a review. Parkinsonism and Related Disorders, v. 10, p. 323-334, 2004. Crossef

VIDRINE, D. J.; AMICK, B. C.; GRITZ, E. R.; ARDUINO, R. C. Funtional status and overall quality of life in a multiethnic HIV-Positive population. Aids Patient Care and STDs, v. 17, n. 4, p. 187-197, 2004. Crossef

WROSH, C.; SCHEIER M. Personality and quality of life: the importance of optimism and goal adjustment. Quality of Life Research, v. 12, n. 1, p. 59-72, 2003. Crossef 